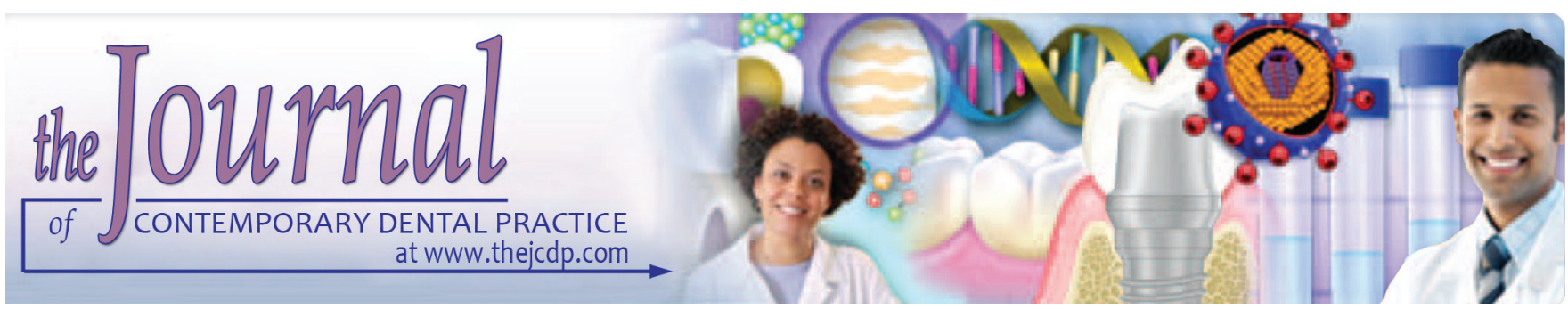

\title{
Comparative Study of Micronuclei Count in Patients with Different Tobacco-related Habits using Exfoliated Buccal Epithelial Cells: A Tool for Assessment of Genotoxicity
}

${ }^{1}$ Kailash C Dash, ${ }^{2}$ Roquaiya Nishat, ${ }^{3}$ Harish Kumar, ${ }^{4}$ Silpiranjan Mishra, ${ }^{5}$ Malvika Raghuvanshi, ${ }^{6}$ Atul Bajoria

\section{ABSTRACT}

Aim: To study and compare the genotoxic effects of tobacco using micronuclei count in individuals with different tobaccorelated habits.

Materials and methods: A cross-sectional study was done comprising 200 individuals, divided into four groups. Group I: 50 subjects with history of tobacco chewing, group II: 50 subjects with a history of smoking tobacco, group III: 50 subjects with a history of both tobacco chewing and smoking, and group IV: 50 subjects without any habits as controls (age-matched). The study groups were individually further divided into three subgroups which comprised of subjects with history of substance abuse for less than 5,5 to 10 , and greater than 10 years. Exfoliated cells from the buccal mucosa of the subjects were collected and stained using Giemsa stain. A total of 1,000 cells were examined for each case and micronuclei frequency was scored according to the guidelines given by Tolbert et al.

Results: The mean number of micronuclei count was $18.28 \pm$ 10.0 in group I (smokeless tobacco users), $11.38 \pm 6.3$ in group II (subjects with history of tobacco smoking), $22.44 \pm 9.8$ in group III (subjects with history of using both smokeless and smokable form of tobacco), and $4.86 \pm 2.4$ in the control group. The statistical difference was found to be highly significant $(p<0.001)$. Similarly, based on the duration, highly significant difference was notable in the mean number of micronuclei in subjects who had a history of substance abuse for more than 10 years.

Conclusion: A significantly higher micronucleus frequency was found in smokeless tobacco users than in smokers and

\footnotetext{
1-3,5Department of Oral Pathology and Microbiology, Kalinga Institute of Dental Sciences, Bhubaneswar, Odisha, India

${ }^{4,6}$ Department of Oral Medicine and Radiology, Kalinga Institute of Dental Sciences, Bhubaneswar, Odisha, India

Corresponding Author: Kailash C Dash, Department of Oral Pathology and Microbiology, Kalinga Institute of Dental Sciences Bhubaneswar, Odisha, India, Phone: +919437902199, e-mail: kcdash1986@gmail.com
}

controls. Micronuclei assay in the exfoliated buccal cells is a useful and minimally invasive method for monitoring early genotoxic damage.

Clinical significance: Micronuclei assay can be used to detect genotoxic damage at the earliest and, if intervened at this point, may prevent frank malignancy, morbidity, and mortality.

Keywords: Genotoxicity, Giemsa stain, Micronuclei, Tobacco.

How to cite this article: Dash KC, Nishat R, Kumar H, Mishra S, Raghuvanshi M, Bajoria A. Comparative Study of Micronuclei Count in Patients with Different Tobacco-related Habits using Exfoliated Buccal Epithelial Cells: A Tool for Assessment of Genotoxicity. J Contemp Dent Pract 2018;19(9):1076-1081.

Source of support: Nil

Conflict of interest: None

\section{INTRODUCTION}

Cancer is a growing epidemic menace and is considered to be one of the major global threats. Around 13 million cancer cases are reported per year worldwide. ${ }^{1}$ Tobacco used in various forms is considered one of the major etiological agents for cancer. In India, 30 to $60 \%$ of total cancers among males and 10 to $30 \%$ among females are tobacco-related. ${ }^{2}$ Oral squamous cell carcinoma affects around 500,000 individuals annually all across the globe and is the most common type of cancer occurring in the oral cavity. ${ }^{3,4}$ In the Indian scenario, the incidence of oral malignancy is 12.6 in 100,000 individuals, which has been on the rise according to recent reports. ${ }^{5}$

Genetic damage lies at the heart of any carcinogenic event. It has been established that genomic damage can be produced by several factors including exposure to genotoxins, medical procedures like radiations or use of certain chemicals, lifestyle factors (tobacco usage and alcohol consumption), and genetic factors. Thus, it is required to have reliable minimally invasive biomarkers which are associated with this genetic damage. ${ }^{6}$ 
Chromosomal aberration investigation, sister chromatid exchange study, and micronucleus test can be used to detect deoxyribonucleic acid (DNA) damage, and hence, evaluate genotoxic effects in tobacco users. Among all these investigations, micronucleus test is found to be more compatible and favorable, as it does not require technique-sensitive and tedious procedures like cell culture, metaphase preparations or DNA-specific staining. Moreover, micronuclei assay is applicable to interphase only, and hence, provides an excellent picture of mitotic interference and chromosomal mutations or breakages, if present. ${ }^{7}$ Thus, micronuclei assay could serve as an excellent potential candidate to be used as a biomarker to detect any genetic damage. ${ }^{6}$

Epithelial cells, in immediate contact with inhaled or ingested genotoxic agents and carcinogens, are most likely to exhibit increased micronuclei frequency. ${ }^{8,9}$ Thus, it can be said that oral epithelial cells which are the first to come in contact with tobacco and its various products might be the preferred site for genotoxic changes and hence, a study of micronuclei assay of these exfoliated epithelial cells could serve as an early biomarker. Moreover, these studies have the advantage of being noninvasive, painless, and also economical. ${ }^{6}$

Micronucleus is an additional small nucleus easily detectable using light microscopy, situated around the main nucleus within the inner half of cytoplasm formed mainly due to chromosomal breakage or due to dysfunction of the mitotic apparatus. ${ }^{8,10,11}$ Micronuclei can either be complete chromosomes or their fragments. ${ }^{12}$ Clastogens can induce chromosomal breaks and yield acentric fragments, which are directly included in the micronuclei. ${ }^{8}$ Alternatively, aneugenic agents prevent formation of mitotic spindle apparatus, due to which whole chromosome lags behind during anaphase. The chromosome is thus surrounded by nuclear envelope forming micronuclei. ${ }^{13}$ In addition, these micronuclei can also be formed due to broken anaphase bridges. ${ }^{14}$

It is a well-known fact that tobacco usage results in genotoxic damage. However, lesser number of studies exist in the literature which have compared the method of tobacco consumption and their effect on genotoxicity. Keeping all these facts in mind, this study was conducted to compare the micronuclei count of exfoliated buccal cells in individuals with different tobacco-related habits. The aim of the present study was to identify and compare the presence of micronuclei in the exfoliated buccal cells in patients with habits of using smokable form of tobacco, chewable form of tobacco, and using both the forms of tobacco and compare with habit-free control.

\section{MATERIALS AND METHODS}

A cross-sectional study was carried out in the Department of Oral Pathology and Microbiology, Kalinga Institute of Dental Sciences, Bhubaneswar, India, after obtaining ethical clearance. Subjects using tobacco either in smoking or smokeless form for a minimum period of 1 year were included in the study. However, subjects with a history of recent viral infection, exposure to potential genotoxic agents including X-rays, chemotherapy, potential occupational exposures, or antibiotic use from past 2 months were excluded from the study, as these factors by virtue of themselves could result in increase in micronuclei count. Moreover, chronic alcoholic subjects with any visible oral pathological lesions, systemic diseases, or subjects using tobacco occasionally or for duration of less than 1 year were also not included, as these can also alter the micronuclei count significantly and hence, would cause erroneous results.

The study population comprised of a total of 200 male subjects aged ranging from 18 to 28 years which was divided into four groups. Group I comprises 50 subjects with a history of tobacco chewing or using smokeless tobacco (paan, gutkha, etc.). Group II comprises 50 subjects with a history of smoking habit. Group III comprises 50 subjects with a history of both tobacco chewing and smoking and group IV comprises 50 subjects without any habits as controls (age-matched). The study groups were individually further divided into three subgroups which comprises subjects with history of substance abuse for less than 5,5 to 10 , and greater than 10 years. Informed written consent was obtained from all the individuals.

\section{Sample Collection, Staining, and Cytological Analysis}

The entire procedure was explained to the patients prior to commencement of the procedure in the language that was best understood by them. After obtaining written consent and performing a routine examination, subjects were asked to rinse their mouth thoroughly with tap water twice to remove debris, excess saliva and reduce the oral bacterial load. Cytological smears were collected from the buccal mucosa using a premoistened wooden spatula. The scraped cells were then transferred onto precleaned labeled glass slides. After air drying the smears, they were immediately placed in $95 \%$ isopropyl alcohol for 20 minutes for fixation and stained using Giemsa stain. The slides were first scanned using low power magnification $(\times 10)$ to determine the quality of staining and number of cells, and then scored for number of micronuclei under 100× magnification (Fig. 1). The zigzag method was followed for screening and counting the micronuclei. A minimum of 1,000 intact cells free of clumping or overlapping were counted for the number of micronuclei, according to the criteria defined by Tolbert et $\mathrm{al}^{15}$ and Holland et al. ${ }^{6}$ 


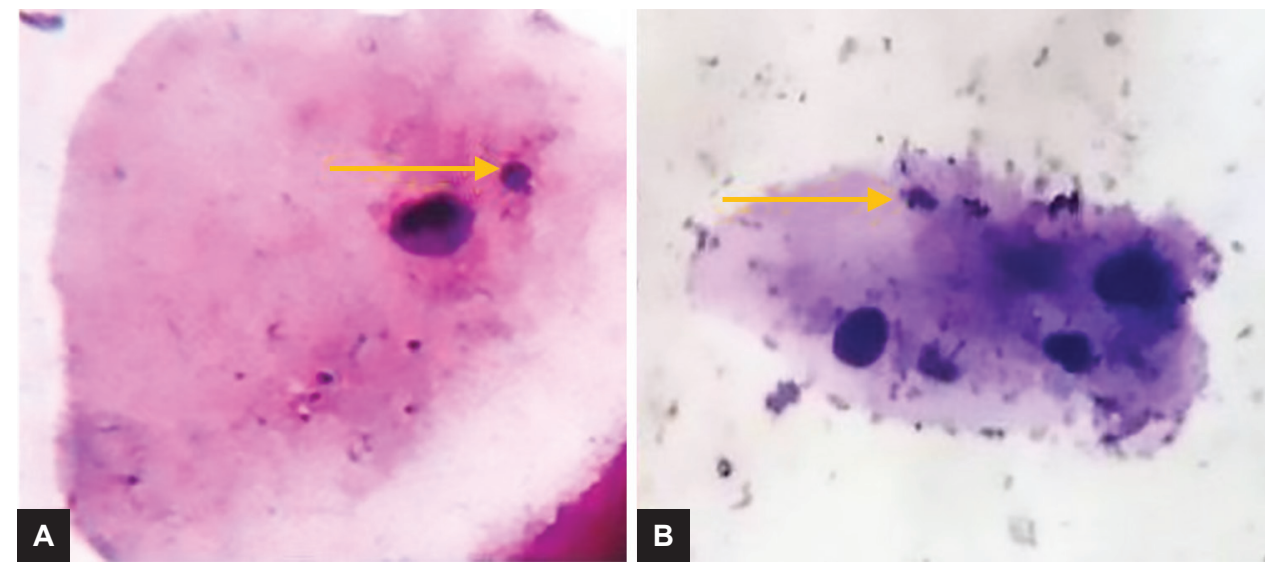

Figs $1 \mathrm{~A}$ and $\mathrm{B}$ : Presence of micronuclei of cytological smear from buccal cells using Giemsa stain and Papanicolaou's stain (40x)

The criteria which were used for identification of micronuclei by Tolbert et al ${ }^{15}$ were: (a) Rounded, smooth perimeter suggestive of a membrane, (b) less than onethird the diameter of associated nucleus, but large enough to discern shape and color, (c) similar staining intensity to that of the nucleus, (d) texture similar to that of nucleus, (e) same focal plane as nucleus, (f) absence of overlap with, or bridge to the nucleus.

The micronuclei frequency and distribution per 1,000 cells were charted separately for patients with smokeless, smoked tobacco habit, patients with habit of usage of both smokeless and smoked tobacco form, and individuals with no deleterious habits. The mean and standard deviation were calculated. Analysis of variance (ANOVA) test was applied. A p-value $(<0.001)$ was considered to be highly significant. Statistical Package for the Social Sciences software version 22.0 was used.

\section{RESULTS}

The results of our study are summarized in Tables 1 to 3 . The frequency of occurrence of micronuclei in exfoliated buccal cells was estimated in different study groups and compared with that of the control group. The mean number of micronuclei count was $18.28 \pm 10.0$ in group I
Table 1: Comparison of mean number of micronuclei in 1,000 cells among four groups (Tobacco chewers, smokers, chewing + smoking, and normal)

\begin{tabular}{llll}
\hline & & \multicolumn{2}{c}{$\begin{array}{c}\text { Number of } \\
\text { micronuclei in } \\
1,000 \text { cells }\end{array}$} \\
\cline { 3 - 4 } Groups (Tobacco habits) & Number & Mean & SD \\
\hline I (chewing) & 50 & 18.28 & 10.000 \\
II (smoking) & 50 & 11.38 & 6.388 \\
III (smoking + chewing) & 50 & 22.44 & 9.846 \\
IV (normal) & 50 & 4.86 & 2.499 \\
Total & 200 & 14.24 & 10.259 \\
ANOVA f-value & 49.105 & & \\
Significance p-value & 0.001 (HS) & & \\
\hline SD: Standard deviation; HS: Highy & & &
\end{tabular}

SD: Standard deviation; HS: Highly significant

(smokeless tobacco users), $11.38 \pm 6.3$ in group II (subjects with history of tobacco smoking), and 22.44 \pm 9.8 in group III (subjects with history of using both smokeless and smokable form of tobacco), and $4.86 \pm 2.4$ in the control group (Table 1). The difference was found to be highly significant $(\mathrm{p}<0.001)$. The mean number of micronuclei found was more in subjects with history of using both smokeless and smokable form of tobacco followed by smokeless tobacco chewers and tobacco smokers.

Table 2: Comparison of mean number of micronuclei in 1,000 cells among three groups according to duration of tobacco habit of chewing

\begin{tabular}{|c|c|c|c|c|c|c|}
\hline \multirow[b]{2}{*}{ Duration } & \multicolumn{2}{|c|}{ Tobacco chewing } & \multicolumn{2}{|c|}{ Tobacco smoking } & \multicolumn{2}{|c|}{ Tobacco chewing + smoking } \\
\hline & Number & Mean $\pm S D$ & Number & Mean $\pm S D$ & Number & Mean $\pm S D$ \\
\hline Group I < 5 years & 12 & $7.33 \pm 1.923$ & 11 & $3.27 \pm 1.555$ & 19 & $12.05 \pm 3.027$ \\
\hline Group II 5-10 years & 23 & $15.65 \pm 2.994$ & 18 & $9.06 \pm 2.127$ & 13 & $23.23 \pm 3.833$ \\
\hline Group III >10 years & 15 & $31.07 \pm 6.617$ & 21 & $17.62 \pm 3.612$ & 18 & $32.83 \pm 4.89$ \\
\hline Total & 50 & $18.28 \pm 10.000$ & 50 & $11.38 \pm 6.388$ & 50 & $22.44 \pm 9.846$ \\
\hline ANOVA f-value & 111.843 & & 106.292 & & 125.79 & \\
\hline Significance $p$-value & $0.001(\mathrm{HS})$ & & $0.001(\mathrm{HS})$ & & $0.001(\mathrm{HS})$ & \\
\hline
\end{tabular}

SD: Standard deviation; HS: Highly significant 
Comparative Study of Micronuclei Count in Patients with Different Tobacco-related Habits

Table 3: Comparison of mean number of micronuclei in 1,000 cells among the subgroups in individual categories based on the duration of habit

\begin{tabular}{|c|c|c|c|c|c|c|}
\hline \multirow[b]{2}{*}{ Groups } & \multicolumn{2}{|c|}{$<5$ years } & \multicolumn{2}{|c|}{$5-10$ years } & \multicolumn{2}{|c|}{$>10$ years } \\
\hline & Number & Mean $\pm S D$ & Number & Mean $\pm S D$ & Number & Mean $\pm S D$ \\
\hline I (chewing) & 12 & $7.33 \pm 1.923$ & 23 & $15.65 \pm 2.994$ & 15 & $31.07 \pm 6.617$ \\
\hline II (smoking) & 11 & $3.27 \pm 1.555$ & 18 & $9.06 \pm 2.127$ & 21 & $17.62 \pm 3.612$ \\
\hline III (smoking + chewing) & 19 & $12.05 \pm 3.027$ & 13 & $23.23 \pm 3.833$ & 18 & $32.83 \pm 4.89$ \\
\hline Total & 42 & $8.4 \pm 4.379$ & 54 & $15.28 \pm 6.101$ & 54 & $26.43 \pm 8.656$ \\
\hline ANOVA f-value & 47.208 & & 86.177 & & 53.590 & \\
\hline Significance $p$-value & 0.001 (HS) & & $0.001(\mathrm{HS})$ & & $0.001(\mathrm{HS})$ & \\
\hline
\end{tabular}

SD: Standard deviation; HS: Highly significant

Further, the study groups were individually divided into three subgroups based on the duration of the habit and statistically analyzed. In group I (smokeless tobacco users), the mean number of micronuclei count in individuals with history of substance abuse for less than 5 years was $7.33 \pm 1.9,15.65 \pm 2.9$ in individuals with abuse history between 5 and 10 years, and $31.07 \pm 6.6$ in individuals with abuse history for more than 10 years. The difference was found to be highly significant (Table 2). In group II (tobacco smokers), the mean number of micronuclei was $3.27 \pm 1.5,9.06 \pm 2.1$, and $17.62 \pm 3.6$ in individuals with history of substance use for $<5$, between 5 and 10, and $>10$ years respectively (Table 2 ). In group III (tobacco smokers and smokeless tobacco users), the mean number of micronuclei in individuals with history of substance use for $<5,5$ to 10 , and $>10$ years was 12.05 $\pm 3.02,23.23 \pm 3.8$, and $32.83 \pm 4.8$ respectively (Table 2 ). The comparisons in groups II and III were also found to be highly significant. Thus, it can be inferred that increased duration of substance use increases the level of genotoxicity and hence, the gradual increase in micronuclei count.

In addition to these findings, highest mean micronuclei count was seen in individuals with combined use of smokeless tobacco and tobacco smokers followed by smokeless tobacco users and then tobacco smokers in individual duration comparison, i.e., in $<5,5$ to 10 , and $>10$ years (Table 3 ). The comparison results were found to be highly significant.

\section{DISCUSSION}

Oral squamous cell carcinoma is a leading cause of morbidity and mortality worldwide with an estimation of 500,000 new cases per year. Tobacco smoking or chewing, alcohol taken in combination or separately are the established factors causing oral cancer. ${ }^{16}$ Genotoxic effects of smoking, chewing betel nut, medical treatment like radiotherapy and occupational exposure to potentially mutagenic and carcinogenic chemicals have been successfully studied using exfoliated buccal cells. ${ }^{17}$ Moreover, cellular alterations like karyolysis, karyorrhexis, micronucleus formation, pyknosis, binucleation, broken egg nucleus, and anucleation can also be screened. ${ }^{18}$ Easy accessibility of tissue for cell sampling, minimally invasive procedure, and no stress to the study subject give the technique an added advantage.

The present study evaluated the mean number of micronuclei in smokeless tobacco users, tobacco smokers, subjects with combined habit of using both smokeless tobacco and smokable form of tobacco and healthy control group. The results showed that the mean number of micronuclei in group III, i.e., subjects with combined habit $(22.44 \pm 9.84)$ was higher as compared with smokeless tobacco users (18.28 \pm 10.00$)$, tobacco smokers (11.38 $\pm 6.38)$, and control group $(4.86 \pm 2.49)$. Similar type of findings has been reported by Patel et $\mathrm{al}^{19}{ }^{19}$ Palaskar and Jindal, ${ }^{20}$ Bansal et al, ${ }^{21}$ Motgi et $\mathrm{al}_{,}{ }^{22}$ and Sangle et al. ${ }^{23}$ According to our results, the extent of genotoxic damage is more in smokeless tobacco users as compared with smoking tobacco. This may be explained by the fact that during chewing, mucosa is in direct contact with tobacco for a longer period of time as compared with smoking which may result in prolonged absorption of nitrosamines, and hence, considerably more nuclear and DNA damage. ${ }^{24}$

In India, individual farmers and companies process smokeless tobacco with varied rate of fermentation and curing, which increases the production of tobacco-specific nitrosamines. ${ }^{22}$ Moreover, smokeless tobacco is generally used in combination with betel leaf, areca nut, or slaked lime, all of which increases the toxicity of tobacco. ${ }^{25}$ Smokers absorb nicotine mainly through the pulmonary vasculature while smokeless tobacco users absorb nicotine through buccal mucosa and gastrointestinal mucosa. ${ }^{22}$ On the contrary, Pradeep et al ${ }^{7}$ reported higher mean micronuclei count in smoking habit followed by smoking with betel quid, smokeless tobacco, and control. This finding has been supported by the fact that since betel leaf is a rich source of antioxidants, it may nullify the genotoxic effects of tobacco. ${ }^{7,26}$

In our study, the highest mean micronuclei count was observed in group III, which shows that the synergistic effect of mixed habit makes the oral mucosal cells more 
vulnerable to genotoxic agents. According to a study done by Znaor et al, ${ }^{27}$ individuals with both the habits of smoking and drinking are 38 times more prone to develop oral cancers than those who do not have any deleterious habit. Prabhakaran and Shaik ${ }^{28}$ estimated the chances of oral cancer to be 7.3 in smokers, 1.3 in alcoholics, and 11.4 in individuals with habit of chewing tobacco. Smokeless tobacco users showed more severe changes as compared with the smoking habit. A positive correlation was found between duration of habit and occurrence of micronuclei in all the groups, the maximum damage being noticed in individuals with habit existing for more than 10 years. Similar result of increase in micronuclei frequency with increased smoking duration was also observed by Naderi et al. ${ }^{29}$

Apart from substance use, micronuclei formation can also be seen in patients with chronic inflammation, chemotherapy, radiation injuries, preneoplastic and neoplastic conditions, genetic diseases like Down syndrome, xeroderma pigmentosum, infective conditions like human papillomavirus infection and metabolic disorders like megaloblastic anemias, and vitamin deficiencies. ${ }^{8}$ Thus, it can be said that micronuclei formation is not always related to genetic damage. However, it can be said that high micronuclei score may be suggestive of genetic damage. Hence, it is important to determine the conditions where micronuclei assay can be used as a tool for assessing genetic damage.

Moreover, accurate identification of micronuclei is equally important, as they may be confused with nuclear debris, bacterial colony, keratohyalin granules, stain deposits, or platelets. In doubtful conditions, DNAspecific stains like Feulgen or acridine orange can be used which are more specific. However, cytokinesis block micronuclei assay is the most popular technique, which can be combined with flow cytometry or fluorescent in situ hybridization technique. ${ }^{8}$ Nevertheless, ordinary routine stains like Giemsa and Papanicolaou's stain can also be used as we have done in the study.

\section{CONCLUSION}

Micronuclei assay is a noninvasive, sensitive, and lowcost technique which can be used as a biomarker to screen genotoxicity. It is evident in the present study that genotoxic damage is associated with tobacco usage, more so in smokeless form. In future, using advanced automated diagnostic and counting devices, these micronuclei may be counted more rapidly and accurately. Moreover, with a larger sample size and matched conditions of the subjects, a baseline may be determined which would be more beneficial in estimating the extent of genotoxic damage.

\section{CLINICAL SIGNIFICANCE}

Micronuclei assay can be used to detect genotoxic damage at the earliest in individuals with history of substance abuse, and, if intervened at this point, may prevent transformation into frank malignancy and hence, reduced morbidity and mortality. Thus, micronuclei assay can serve as an excellent biomarker for genotoxicity.

\section{REFERENCES}

1. Jemal A, Bray F, Center MM, Ferlay J, Ward E, Forman D. Global cancer statistics. CA Cancer J Clin 2011 Mar-Apr;61(2):69-90.

2. NCDIR. Three year report of population based and hospital based cancer registries (2012-2014). Bengaluru: NCDIR; 2016. Available from: http:/ / www.ncdirindia.org/.

3. Johnson NW, Warnakulasuriya S, Gupta PC, Dimba E, Chindia M, Otoh EC, Sankaranarayanan R, Califano J, Kowalski L. Global oral health inequalities in incidence and outcomes for oral cancer: causes and solutions. Adv Dent Res 2011 May;23(2):237-246.

4. Sousa MC, Alves MG, Souza LA, Brandao AA, Almeida JD, Cabral LA. Correlation of clinical, cytological and histological findings on oral squamous cell carcinoma. Oncol Lett 2014 Aug;8(2):799-802.

5. Misra C,Majumder M, BajajS, GhoshS, Roy B, RoychoudhuryS. Polymorphism at p53, p73, and MDM2 loci modulate the risk of tobacco associated leukoplakia and oral cancer. Mol Carcinog 2009 Sep;48(9):790-800.

6. Holland N, Bolognesi C, Kirsch-Volders MC, Bonassi S, Zeiger E, Knasmueller S, Fenech M. The micronucleus assay in human buccal cells as a tool for biomonitoring DNA damage: the HUMN project perspective on current status and knowledge gaps. Mutat Res 2008 Jul-Aug;659(1-2):93-108.

7. Pradeep MR, Guruprasad Y, Jose M, Saxena K, Deepa K, Prabhu V. Comparative study of genotoxicity in different tobacco related habits using micronucleus assay in exfoliated buccal epithelial cells. J Clin Diagn Res 2014 May;8(5):ZC21-ZC24.

8. Samanta S, Dey P. Micronucleus and its application. Diagn Cytopathol 2010 Jan;40(1):84-90.

9. Kimura M, Umegaki K, Higuchi M, Thomas P, Fenech M. Methylenetetrahydrofolate reductase C677T polymorphism, folic acid and riboflavin are important determinants of genome stability in cultured human lymphocytes. J Nutr 2004 Jan;134(1):48-56.

10. Fenech M, Chang WP, Kirsch-Volders M, Holland N, Bonassi S, Zeiger E. Human Micronnucleus project. HUMN project: detailed description of the scoring criteria for the cytokinesis-block micronucleus assay using isolated human lymphocyte cultures. Mutat Res 2003 Jan;534(1-2):65-75.

11. Falck GC, Catalan J, Norppa H. Nature of anaphase laggards and micronuclei in female cytokinesis blocked lymphocytes. Mutagenesis 2002 Mar;17(2):111-117.

12. Jain V, Lohra P, Priya B, Sindhu D. Buccal cell micronuclei assay: a non invasive genotoxic marker. Int J Contemp Med Res 2017 Jan;4(1):100-104.

13. Cimini D, Fioravanti D, Salmon ED, Degrassi F. Merotelic kinetochore orientation versus chromosome mono orientation in the origin of lagging chromosomes in human primary cells. J Cell Sci 2002 Feb;115(Pt 3):507-515. 
14. Saunders WS, Shuster M, Huang X, Gharaibeh B, Enyenihi AH, Petersen I, Gollin SM. Chromosomal instability and cytoskeletal defects in oral cancer cells. Proc Natl Acad Sci U S A 2000 Jan;97(1):303-308.

15. Tolbert PE, Shy CM, Allen JW. Micronucleus and other nuclear anomalies in buccal smears: method development. Mut Res 1992 Feb;271(1):69-77.

16. Katarkar A, Mukherjee S, Khan MH, Ray JG, Chaudhuri K. Comparative evaluation of genotoxicity by micronucleus assay in the buccal mucosa over comet assay in peripheral blood in oral precancer and cancer patients. Mutagenesis 2014 Sep;29(5):325-334.

17. Jyoti S, Siddique YH, Khan S, Naz F, Rahul Ali F. Effect on micronucleus frequency and DNA damage in buccal epithelial cells of various factors among pan masala and gutkha chewers. Oral Sci Int 2015 Jan;12(1):9-14.

18. Jois HS, Kale AD, Mohan Kumar KP. Micronucleus as a potential biomarker of oral carcinogenesis. Indian J Dent Adv 2010 Jan;2:1-5.

19. Patel BP, Trivedi PJ, Brahmbhatt MM, Shukla SN, Shah PM, Bakshi SR. Micronuclei and chromosomal aberrations in healthy tobacco chewers and controls: a study from Gujarat, India. Arch Oncol 2009 Jul;17(1-2):7-10.

20. Palaskar S, Jindal C. Evaluation of micronuclei using Papanicolaou and may Grunwald-Giemsa stain in individuals with different tobacco habits: a comparative study. J Clin Diagn Res 2011 Dec;4(6):3607-3613.

21. Bansal H, Sandhu VS, Bhandari R, Sharma D. Evaluation of micronuclei in tobacco users: a study in Punjabi population. Contemp Clin Dent 2012 Apr;3(2):184-187.

22. Motgi AA, Chavan MS, Diwan NN, Chowdhery A, Channe PP, Shete MV. Assessment of cytogenetic damage in the form of micronuclei in oral epithelial cells in patients using smokeless and smoked form of tobacco and non tobacco users and its relevance for oral cancer. J Cancer Res Ther 2014 Apr;10(1):165-170.

23. Sangle VA, Bijjaragi S, Shah N, Kangane S, Ghule HM, Rani SA. Comparative study of frequency of micronuclei in normal, potentially malignant diseases and oral squamous cell carcinoma. J Nat Sci Bio Med 2016 Jan-Jun;7(1):33-38.

24. Proia NK, Paszkiewicz GM, Nasca MA, Franke GE, Pauly JL. Smoking and smokeless tobacco-associated human buccal cell mutations and their association with oral cancer-a review. Cancer Epidemiol Biomarkers Prev 2006 Jun;15(6): 1061-1077.

25. Rodu B, Jansson C. Smokeless tobacco and oral cancer: a review of the risks and determinants. Crit Rev Oral Biol Med 2004 Sep;15(5):252-263.

26. Rathee JS, Patro BS, Mula S, Gamre S, Chattopadhyay S. Antioxidant activity of Piper betel leaf extract and its constituents. J Agric Food Chem 2006 Nov;54(24):9046-9054.

27. Znaor A, Brennan P, Gajalakshmi V, Mathew A, Shanta V, Varghese C, Boffetta P. Independent and combined effects of tobacco smoking, chewing and alcohol drinking on the risk of oral, pharyngeal and esophageal cancers in Indian men. Int J Cancer 2003 Jul;105(5):681-686.

28. Prabhakaran, PS.; Shaik, HA. Estimation of oral cancer: oral oncology CME annual. Bengaluru: Kidwai Memorial Institute of Oncology; 2002. pp. 1-10.

29. Naderi NJ, Farhadi S, Sarshar S. Micronucleus assay of buccal mucosa cells in smokers with the history of smoking less and more than 10 years. Indian J Pathol Microbiol 2012 Oct-Dec;55(4):433-438. 